\title{
Conspirations des réseaux plats Dessiner des visualisations sur une surface sphérique continue
}

\author{
Dario Rodighiero (Harvard University)
}

RÉSUMÉ - Malgré la grande littérature concernant les visualisations de réseau, leur représentation graphique n'est guère un objet d'investigation. Parfois, cela mérite plus d'attention, surtout lorsque des individus sont représentés. Traduire visuellement des communautés en réseau, par exemple, implique que certains individus soient positionnés aux frontières de la représentation. Cette hypothèse apparaît injuste, surtout si chaque individu de la communauté est connecté avec tout le monde.

MOTS-CLÉS - Graphes, Réseaux sociaux, Centralité, Démocratie, Projections

Cet article est publié dans la série Études digitales, dans un numéro spécial dirigé par Éric Guichard, imprimé en 2022 par les Classiques Garnier. Il s'agit de la version pré-imprimée soutenue par le Fonds national suisse (FNS) qui finance le projet de recherche numéro P400PS_194442. L'article a été édité à partir de la version anglaise, précédemment présentée à la 24e conférence internationale sur la visualisation de l'information en 2020.

L'article peut être cité en français ou dans sa version originale en anglais de cette manière :

Rodighiero, Dario. 2022. "Conspirations des réseaux plats : dessiner des visualisations sur une surface sphérique continue.” Edited by Éric Guichard. Études digitales, cartographie et visualisation, 2020-2 (10): 197-211.

https://doi.org/10.48611/ISBN.978-2-406-12722-2.P.0197.

Rodighiero, Dario. 2020. “Drawing Network Visualizations on a Continuous, Spherical Surface.” In 24th International Conference Information Visualisation (IV), 574-80. Melbourne and Vienna: IEEE. https://doi.org/10/ghmntz. 
La visualisation de données est née au début $d u x^{e}$ siècle. Bien que son histoire remonte à une époque plus ancienne où les cartes étaient faites à la main, les praticiens actuels ne semblent pas curieux de cet héritage, préférant les techniques contemporaines. Pourtant, il ne fait aucun doute qu'un retour sur l'histoire de la visualisation serait fécond. Cet article se penchera d'ailleurs sur les apports de la cartographie, l'une des disciplines qui ont contribué à façonner le langage visuel des données.

Les visualisations de réseaux sont généralement dessinées sur une surface plane, ce qui les rend imprimables et portables comme des cartes ${ }^{1}$. Malgré ces avantages apparaît un inconvénient majeur lorsqu'un groupe d'individus est représenté : une partie d'entre eux apparaît sur les bords, loin des autres. C’est une caractéristique des réseaux planaires, qui sont couramment produits.

Lors de deux événements au cours desquels des « communautés » scientifiques ont été ainsi représentées, cet effet de la centralité était facile à remarquer. Les spectateurs de tels graphes étaient satisfaits lorsqu'ils apparaissaient au centre du réseau, et mécontents lorsque leur nom était placé sur les bordures. Au-delà de toute mesure potentielle pouvant réguler l'agencement d'un réseau, il semble injuste de prédestiner quelques individus aux frontières de la visualisation. Pour pondérer ce déterminisme métrologique, l’idée est de représenter les réseaux par la visualisation sphérique, que la Terre exprime bien dans une approche topologique sans bords. Telle surface peut représenter plus « démocratiquement » la visualisation en réseau.

Les réseaux sphériques sont dépliés afin de les rendre lisibles, en utilisant des techniques de cartographie géographique telles que les projections Mercator ou quinconciale. La variété de ces techniques est ensuite appliquée à un ensemble de données représentant le collectif scientifique des humanités numériques. La distance lexicale entre les conférenciers est enfin visualisée de façon que la fonctionnalité s'efface au profit d'un sens esthétique².

\section{VIVRE DANS UN RÉSEAU PLAT}

En 2018, se diffusait un documentaire sur la Flat Earth Society intitulé « Behind the Curve ${ }^{3}$ ». Cette organisation prétend que la sphéricité de la Terre n'est pas perceptible par les humains et donc que l'expérience rend intenable l'idée de la sphéricité de notre planète. De plus, ce groupe affirme que le gouvernement américain a monté un complot pour que ses citoyens restent dociles et ignorants à ce sujet ${ }^{4}$. Bien que la science fournisse suffisamment d'informations pour

\footnotetext{
${ }^{1}$ Latour, Bruno. 1986. "Visualisation and Cognition: Thinking with Eyes and Hands.” Edited by Henrica Kuklick. Knowledge and Society Studies in the Sociology of Culture Past and Present 6: 1-40.

${ }^{2}$ Manovich, Lev. 2008. "Data Visualization as New Abstraction and Anti-Sublime." In Small Tech: The Culture of Digital Tools, edited by Byron Hawk, David M. Rieder, and Ollie O. Oviedo. Electronic Mediations 22. University of Minnesota Press.

${ }^{3}$ Clark, Daniel J. 2018. Behind the Curve. https://www.imdb.com/title/tt8132700/.

${ }^{4}$ Richardson, Gary. 2019. "It's a Flat Earth.” The New Yorker, April 1, 2019. https://www.newyorker.com/magazine/2019/04/08/its-a-flat-earth.
} 
contredire cette étrange théorie, le mouvement des Flat Earthers peut au moins nous stimuler intellectuellement: ce groupe a l'audace de remettre en question une hypothèse que tout le monde tient pour acquise.

À l'école et au lycée, nous apprenons diverses choses,comme la théorie de l'évolution ou la théorie du Big Bang. Au fil des ans, nous développons une pensée critique, une heuristique et des méthodes personnelles pour éprouver leur véracité. La pensée critique peut suivre des chemins complexes et délicats, y compris dans la communauté scientifique ${ }^{5}$. Nier l'évidence, comme le font les flat-earthers, nous permet parfois de voir un problème sous un autre angle. La représentation planaire des graphes et des réseaux sollicite-t-elle des a priori qui pourraient avoir des analogies avec les préjugés de la Flat Earth Society? Le postulat d'une planéarité des réseaux, pourtant contredit par diverses méthodes des sciences sociales appuyées par des représentations graphiques, semble largement sollicité 6 . Nous voudrions ici illustrer l'avantage de penser dans un espace non plus euclidien, mais riemannien.

\section{LA DIFFICULTÉ DE LA REPRÉSENTATION}

Les peintres savent qu'il n'est pas facile de représenter quelqu'un. Cela exige un mélange d'aptitudes telles que le goût, la délicatesse et les capacités techniques. Même lorsque ces qualités excellent, il peut arriver que le sujet ne soit pas d'accord avec la représentation, comme ce fut le cas pour Winston Churchill : pour célébrer son $80^{\mathrm{e}}$ anniversaire, la Chambre des Communes et la Chambre des Lords firent appel au peintre anglais Graham Sutherland pour en faire le portrait. Churchill en fut si peu satisfait que la toile a disparu de sa maison de campagne ${ }^{7}$. Il ne nous en reste qu'une esquisse de son visage, à la National Portrait Gallery.

Cette anecdote montre la complexité de la représentation de l'identité personnelle. Avec les médias contemporains, elle ouvre sur la question de la représentation d'individus moins célèbres, du fait de la récente facilité à quantifier nos ego à partir de données en ligne ${ }^{8}$. De telles représentations posent divers problèmes, y compris éthiques, car elles concernent désormais presque tous les êtres humains, sans qu'ils aient leur mot à dire. Pour autant, le débat scientifique sur la représentation des individus à partir de leurs « données personnelles », à quelques exceptions près ${ }^{9}$, reste embryonnaire.

\footnotetext{
${ }^{5}$ Kuhn, Thomas S. 1962. The Structure of Scientific Revolutions. University of Chicago Press.

${ }^{6}$ Barabási, Albert-László, and Márton Pósfai. 2016. Network Science. Network science: Cambridge University Press.

${ }^{7}$ Clubbe, John. (2005) 2016. Byron, Sully, and the Power of Portraiture. Second Edition. New York: Routledge. https://doi.org/10.4324/9781315619798.

${ }^{8}$ Rodighiero, Dario, and Loup Cellard. 2019. “Self-Recognition in Data Visualization.” EspacesTemps.Net Electronic Journal of Humanities and Social Sciences, August. https://doi.org/10/ghmsrc.

${ }^{9}$ Floridi, Luciano. 2019. The Logic of Information: A Theory of Philosophy as Conceptual Design. First edition. Oxford; New York: Oxford University Press.
} 


\section{LA QUESTION DE LA CENTRALITÉ DES RÉSEAUX}

Lors de la conférence en humanités numériques de $2014^{10}$, quand les universitaires ont été invités à se reconnaître dans une visualisation en réseau représentant leur communauté scientifique, les réactions ont été fort variées. Certains étaient offensés de ne pas figurer dans la visualisation, d'autres étaient heureux d'y être, signalant leur bonheur via Twitter ${ }^{11}$. Des réactions analogues ont été repérées à l'occasion de la journée de la recherche de la Faculté de l'environnement naturel, architectural et construit à l'EPFL ${ }^{12}$.

Souvent, la plupart des récriminations étaient liées à des problèmes résolubles en fouillant un peu plus les données ; mais un autre aspect n'était pas complètement ciblé. Après réflexion, il parut évident que la réaction des enquêtes était liée à la position de leur représentation : plus une personne était placée au centre, plus elle se sentait flattée.

Ceci renvoie au fonctionnement de la mesure de centralité, qui détermine la pertinence du nœu ${ }^{13}$. La centralité peut être calculée mathématiquement, mais aussi interprétée visuellement, car dans les réseaux, il est assez facile de voir si un nœud est central ou non. Le résultat graphique ne correspond pas toujours aux attentes des personnes représentées, qui attendent souvent d'avoir la preuve qu'elles sont populaires et dignes d'attention.

\section{À LA RECHERCHE D’UN ESPACE CONTINU}

Placer des individus à la périphérie des réseaux semble raisonnable : cela renvoie à une caractéristique que les réseaux « incarnent » lorsqu'ils sont projetés sur une surface plane. Placer quelques nœuds aux marges du réseau est une règle que la majorité des logiciels et des bibliothèques suivent, et qui peut avoir quelques fondements mathématiques, sinon géométriques.

Lorsque les entités du réseau ne sont pas humaines, il n'y a pas d'impact significatif. Mais lorsque les individus sont les nœuds du réseau, le fait d'être en marge peut générer un état de mécontentement: un peu comme dans la société réelle, quand les individus peuvent se sentir découragés lorsqu'ils sont marginalisés. Le fait de placer quelqu'un à la périphérie d'un réseau peut être assimilé à une forme d'exclusion, même si d'aucuns, comme Évariste Galois, se souciaient peu de telles singularisations. Une des causes de cette marginalisation graphique et donc peut-être sociale est liée à la mesure de la centralité.

\footnotetext{
${ }^{10}$ Rigal, Alexandre, and Dario Rodighiero. 2015. “Trajectoire d'une représentation cartographique en réseau.” Edited by Armelle Couillet Jasmine Desclaux-Salachas. Cartes \& Géomatique 225: 33-41. https://infoscience.epfl.ch/record/214582/.

${ }^{11}$ Rodighiero, Dario. 2018. "Printing Walkable Visualizations." In Proceedings of the 5th Biennial Research Transdisciplinary Imaging Conference, 58-73. Figshare. https://doi.org/10/gfvs3w.

${ }^{12}$ Rodighiero, Dario, Frédéric Kaplan, and Boris Beaude. 2018. "Mapping Affinities in Academic Organizations.” Frontiers in Research Metrics and Analytics 3 (4). https://doi.org/10/ghmsrg.

${ }^{13}$ Scott, John. (1991) 2000. Social Network Analysis: A Handbook. Second edition. London and Thousands Oaks, CA: SAGE Publications.
} 
Hélas, il n'y a aucun moyen de se débarrasser de la centralité tant que les réseaux sont dessinés sur une surface plane. La question est donc simple : est-il possible de concevoir l'espace représenté sans centralité ? Oui, c'est possible, à condition de se débarrasser des cadres picturaux qui imposent la limite de l'espace. Comment produire des représentations visuelles qui les rendent inutiles? Nous en connaissons une : celle induite par le globe terrestre, qui « casse » le cadre du planisphère. En utilisant la sphéricité de la Terre pour visualiser les liens sociaux entre les individus d'une manière moins biaisée, la mesure de la centralité sera remplacée par celle de la densité.

\section{SOCIABILITÉS LINGUISTIQUES}

La centralité s'avère pertinente sous réserve de deux conditions : (1) les nœuds correspondent à des individus qui font partie d'un groupe et (2) un degré élevé de connectivité caractérise ce groupe. Ce qui fonctionne assez bien pour des communautés relativement petites comme celles du monde académique, qui sont fréquemment sollicitées pour repérer des clusters thématiques, souvent sur une base bibliographique à l'intérieur des disciplines comme la bibliométrie.

Pour autant, les communautés scientifiques sont construites sur des liens à multiples facettes. Les chercheurs sont en relation les uns avec les autres parce qu'ils enseignent, publient et conseillent avec leurs pairs, ce qui crée un tissu multidimensionnel entrelacé dont la complexité est proportionnelle à la diversité des pratiques. Parmi toutes les mesures potentielles qu'un analyste peut employer pour représenter la communauté scientifique à l'aide d'un réseau, la langue représente un choix intéressant pour de nombreuses raisons : elle diffère du système de citation, car elle est une caractéristique largement partagée au-delà de tout rôle ou ancienneté. Elle est à la base de la communication et n'est soumise à aucun droit d'auteur. Une visualisation de réseau basée sur une telle métrique renforce alors les indices de démocratie, d'égalité et d'impartialité.

Une conférence scientifique, par exemple, est un cadre qui remplit ces deux conditions : (1) une conférence est un groupe composé d'individus et (2) le vocabulaire qu'ils utilisent donne vie à une structure dense de connexions. Les documents des conférences peuvent être analysés en termes de fréquence pour calculer une métrique. L'analyse de texte peut aider à créer cette distance lexicale, qui peut ensuite être traduite en un graphique où les individus sont liés par une terminologie commune.

\section{L’ÉTUDE DE CAS DE DH2019}

Les participants à un grand colloque s'orientent dans ses diverses sessions en utilisant un guide qui illustre quand et où quelqu'un parle. Bien que ce système soit bien établi, il offre une compréhension limitée et fragmentée : par page. Des approches plus récentes visent à décrire 
son intégralité à l'aide d'instruments numériques, comme dans le cas de la cartographie lexicale ${ }^{14}$.

La cartographie lexicale est basée sur la fréquence des mots, qui peut être calculée à partir des textes écrits par les conférenciers. Une telle métrique peut être basée sur différentes méthodes statistiques telles que l'algorithme TF-IDF, qui calcule la fréquence des mots pour chaque auteur, divisée ensuite par la fréquence du corpus entier. Le résultat est un classement, pour chaque auteur, des mots les plus pertinents triés par importance. Tous les mots partagés entre deux chercheurs deviennent des liens qui décrivent la structure du réseau.

L'étude de la conférence en humanités numériques qui s'est tenue à Utrecht (DH2019) confirme la question de la centralité soulevée dans ces pages. Malgré la forte connectivité des liens lexicaux, un ensemble d'auteurs était confiné vers les frontières du réseau, bien qu'aucun de ces auteurs ne méritât d'être éloigné du centre (voir figure 1). Bien que le lexique soit la langue partagée qui rassemble le collectif scientifique, une forme de discrimination visuelle sépare les conférenciers au centre depuis les collègues plus éloignés dans un espace qui manque de continuité.

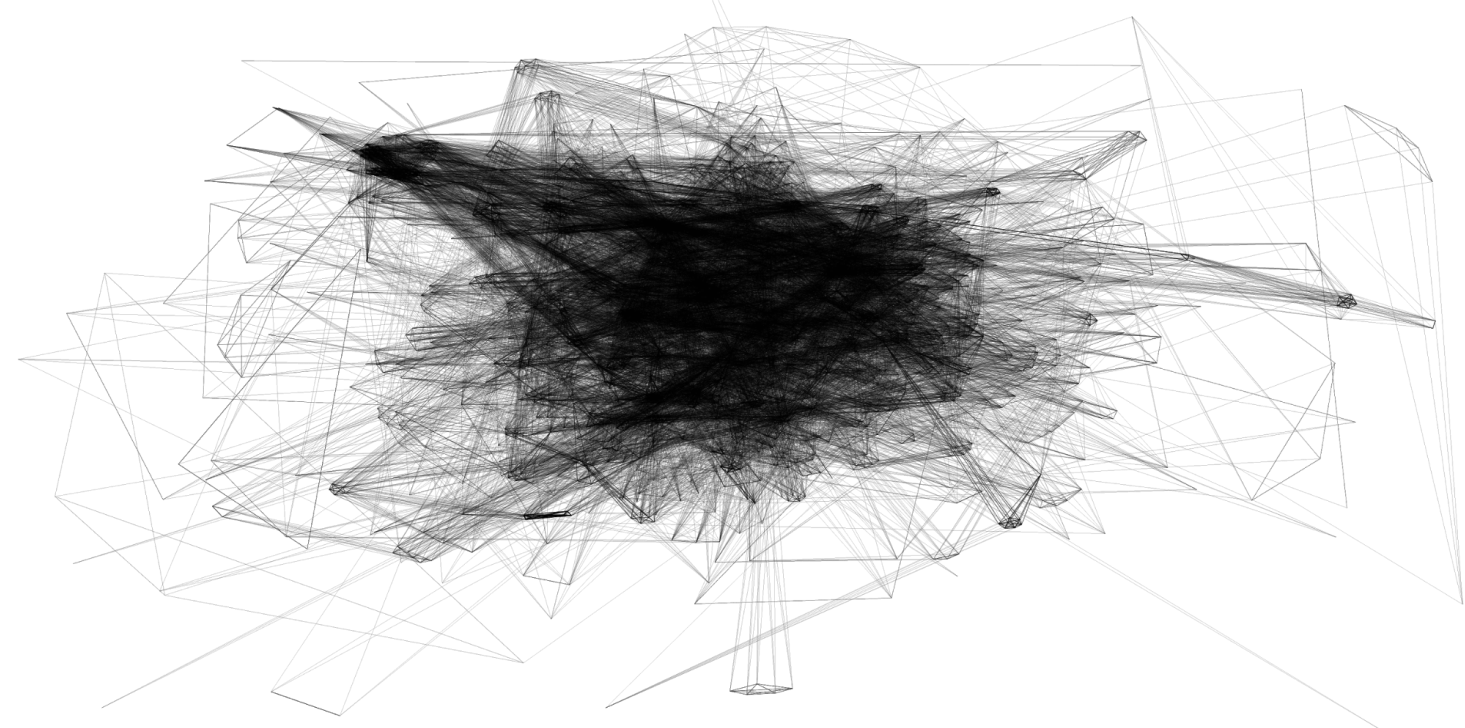

Fig. 1 - Cette visualisation de réseau représente la conférence sur les humanités numériques qui a eu lieu en 2019 à Utrecht, aux Pays-Bas. La visualisation est composée de conférenciers reliés par leur terminologie commune. Bien que la connectivité soit élevée, certains chercheurs sont de toute façon placés sur les bords, transformant le réseau en une sorte de classement.

\footnotetext{
${ }^{14}$ Moon, Chloe Ye Eun, and Dario Rodighiero. 2020. "Mapping as a Contemporary Instrument for Orientation in Conferences." In Atti Del IX Convegno Annuale AIUCD. La Svolta Inevitabile: Sfide e Prospettive per l'informatica Umanistica. Zenodo. https://doi.org/10.5281/zenodo.3611340.
} 


\section{DESSINER SUR LA SURFACE SPHÉRIQUE}

L’organisation d'un réseau est basée sur des méthodes mathématiques qui simulent la dynamique moléculaire. L'intégration de Verlet est une méthode qu'Isaac Newton a utilisée pour intégrer les équations des mouvements, mais qui est aujourd'hui employée dans la mise en forme de graphes dirigés par la force. En utilisant un ensemble de données relationnelles où des bords pondérés relient les nœuds, le réseau peut être représenté sur une surface bidimensionnelle, ce qui rend sa structure lisible. D3 est l'une des bibliothèques gratuites disponibles pour dessiner des réseaux en utilisant JavaScript $t^{15}$.

L'agencement de réseau est le résultat d'un processus de stabilisation dans lequel la force de liens est utilisée dans un processus de négociation spatiale. Contrairement à la disposition des forces appliquées pour obtenir un réseau plat, la conception d'un réseau tridimensionnel nécessite un système de forces capable de tracer le réseau dans un espace tridimensionne ${ }^{16}$. De plus, dessiner sur une surface sphérique nécessite une force supplémentaire au système, agissant de manière semblable à la gravité. Une telle force fait en sorte que les nœuds, qui rebondissent dans l'espace pendant la disposition (voir figure 2), sont attirés par une surface sphérique invisible autour du centre.

La somme de ces forces fixe les nœuds sur la surface sphérique, mais ce n'est pas la seule condition. Les paramètres jouent un rôle important dans les réseaux sphériques. La couverture optimale de la surface résulte d'un équilibre délicat entre deux paramètres qui agissent sur le système : l'intensité de la force de liaisons et de la force many-body appliquée à tous les nœuds. Lorsque ces forces ne sont pas correctement équilibrées, le risque est d'obtenir un réseau rond et légèrement courbé qui ressemble à la forme de la plaque antarctique. Si cela devait arriver, la force de liaison serait relâchée pour faire glisser doucement le réseau sur la sphère jusqu'à ce qu'il soit entièrement recouvert. De même, la force de plusieurs corps doit être augmentée pour détourner tout éventuel groupe de nœuds, ce qui pourrait rendre la visualisation illisible.

\footnotetext{
${ }^{15}$ Bostock, Michael, Vadim Ogievetsky, and Jeffrey Heer. 2011. “D3: Data-Driven Documents.” IEEE Transactions on Visualization and Computer Graphics 17 (12): 2301-9. https://doi.org/10/b7bhhf.

${ }^{16}$ Le modèle tridimensionnel de Vasco Asturiano est téléchargeable sur https://github.com/vasturiano/d3-force-3d.
} 

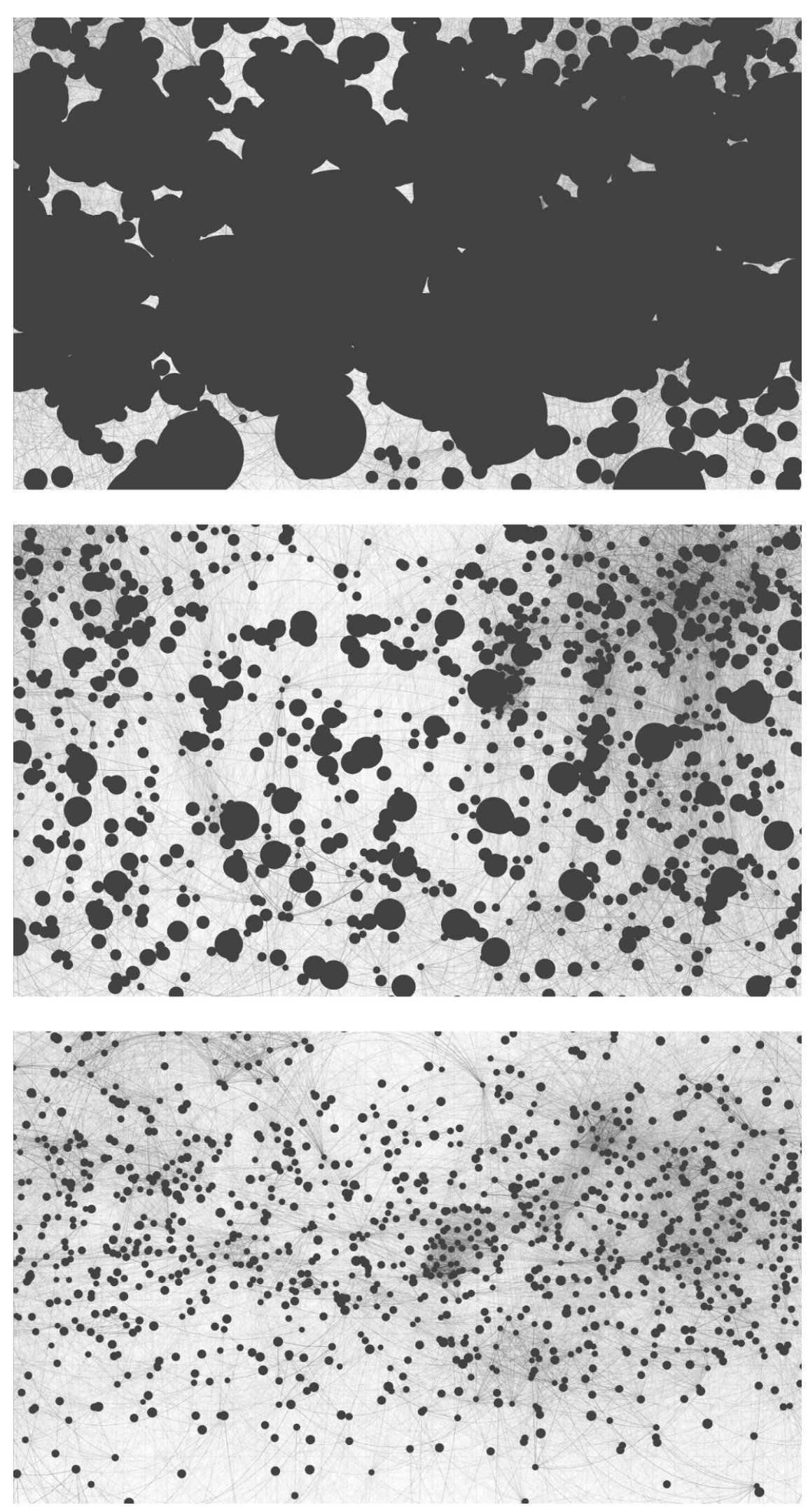

Fig. 2 -Cette séquence montre trois moments de l'arrangement du réseau. On remarque notamment que la taille des noeuds change en fonction de leur position : les noeuds les plus larges sont plus proches $d u$ spectateur. Au cours de l'arrangement, ces nouds adoptent la même taille, ce qui signifie qu'ils s'organisent sur la surface sphérique à la même distance du centre. 
Le résultat de l'agence est une composition de nœuds qui dessine une sphère (voir figure 3). La ressemblance avec un globe est encore renforcée par les lignes courbes qui courent sur la surface. Les 1000 nœuds et les 140000 liens forment un réseau dense qui conduit à des résultats favorables à un arrangement sphérique.

Le réseau sphérique ne peut pas être lu en matière de centralité. Une telle mesure est absente au profit d'une autre, la densité. Une densité haute du réseau correspond à une zone marquée par le nombre de nœuds, qui indique un vocabulaire très partagé, comme dans le cas d'un article de conférence coécrit. Lorsque des auteurs sont isolés dans des zones plus raréfiées, ils se trouvent toujours dans un quartier contextuel à 360 degrés, malgré la distance qui peut les séparer des autres.

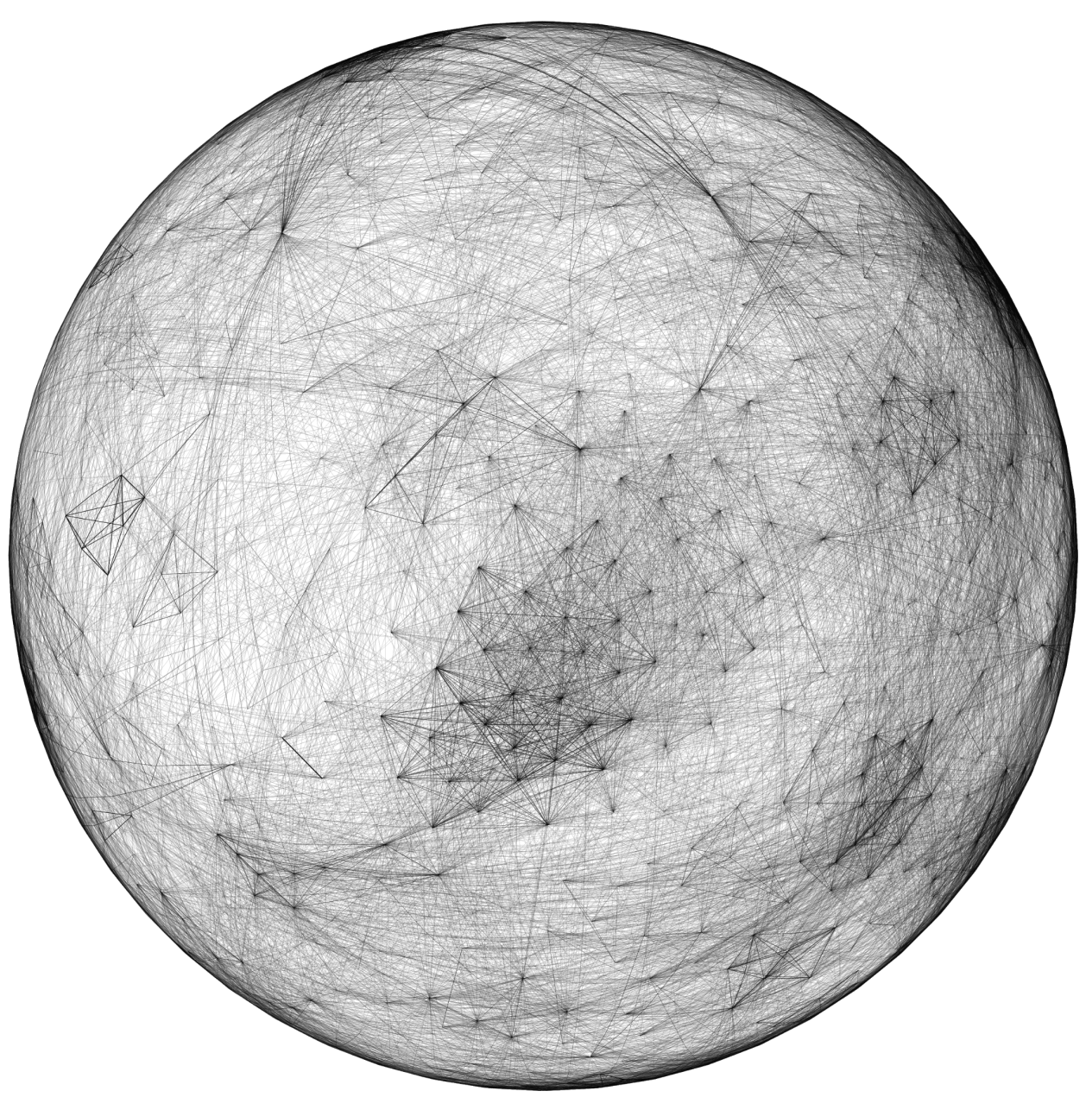

Fig. 3 - Cet instantané montre une représentation de la conférence des humanités numériques 2019. Le réseau plat présenté dans la figure 1 est disposé différemment sur une surface sphérique. Aucun individu n'est placé sur les frontières. Dans une telle condition, il n'est pas possible de créer un classement basé sur la centralité. 


\section{PROJECTION CARTOGRAPHIQUE DES RÉSEAUX SPHÉRIQUES}

La projection cartographique est l'un des gestes humains les plus ambitieux. Elle incarne l'effort gigantesque pour saisir et représenter le volume de la Terre. Il est possible d'appliquer cette projection à la visualisation sphérique de réseau pour réintroduire la lisibilité de la surface planaire, précédemment perdue avec l'agencement tridimensionnel. La moitié cachée de la sphère devient alors visible. Ce traitement est techniquement réalisable grâce à la composante géographique de $\mathrm{D} 3^{17}$ qui permet d'étendre la bibliothèque $\mathrm{D} 3$ avec différentes projections cartographiques.

L'avantage d'une meilleure lisibilité de la totalité du réseau sphérique est contrebalancé par des inconvénients, comme c'est le cas dans de nombreux processus de transformation. Le principal inconvénient est la déformation des distances entre les nœuds, clairement visible dans la projection de Mercator ${ }^{18}$ qui montre une déformation proportionnelle à la distance par rapport à l'équateur. La projection de Mercator est l'une des principales projections, car elle maintient la proportion correcte dans de nombreuses zones peuplées. Cependant, la déformation de la Norvège est clairement perceptible par rapport à la grille ainsi que la fausse magnitude de l'Antarctique.

Les projections de réseau doivent donc être interprétées avec prudence, car, comme une carte, les distances peuvent être représentées de manière trompeuse. Comme pour toutes les visualisations de données, il est nécessaire d'avoir une conscience générale du processus de création pour comprendre chaque résultat. Une telle connaissance permettra au lecteur de lire les supports visuels avec les précautions nécessaires.

Les résultats de l'application de la projection Mercator au DH2019 sont présentés à la figure 5, qui nous donne une idée de la déformation du réseau, en particulier en haut et en bas de l'image où les connexions sont plutôt espacées. Il faut également considérer que ces marges sont définies par une intervention manuelle, car la projection Mercator va à l'infini vers le Nord et le Sud. En outre, il existe une autre différence par rapport à une projection géographique standard : le territoire n'a pas de formes géométriques régulières. Les lignes qui sont droites sur la surface sphérique donnent une idée de la déformation du plan. La figure 4 montre comment les lignes, qui sont soigneusement réparties sur la surface sphérique, ont tendance à être plus courbées en s'éloignant de l'équateur.

\footnotetext{
${ }^{17}$ Plus d'informations sur les projections géographiques D3 sur https://github.com/d3/d3-geo-projection.

${ }^{18}$ Monmonier, Mark S. 2004. Rhumb Lines and Map Wars: A Social History of the Mercator Projection. Chicago: University of Chicago Press.
} 


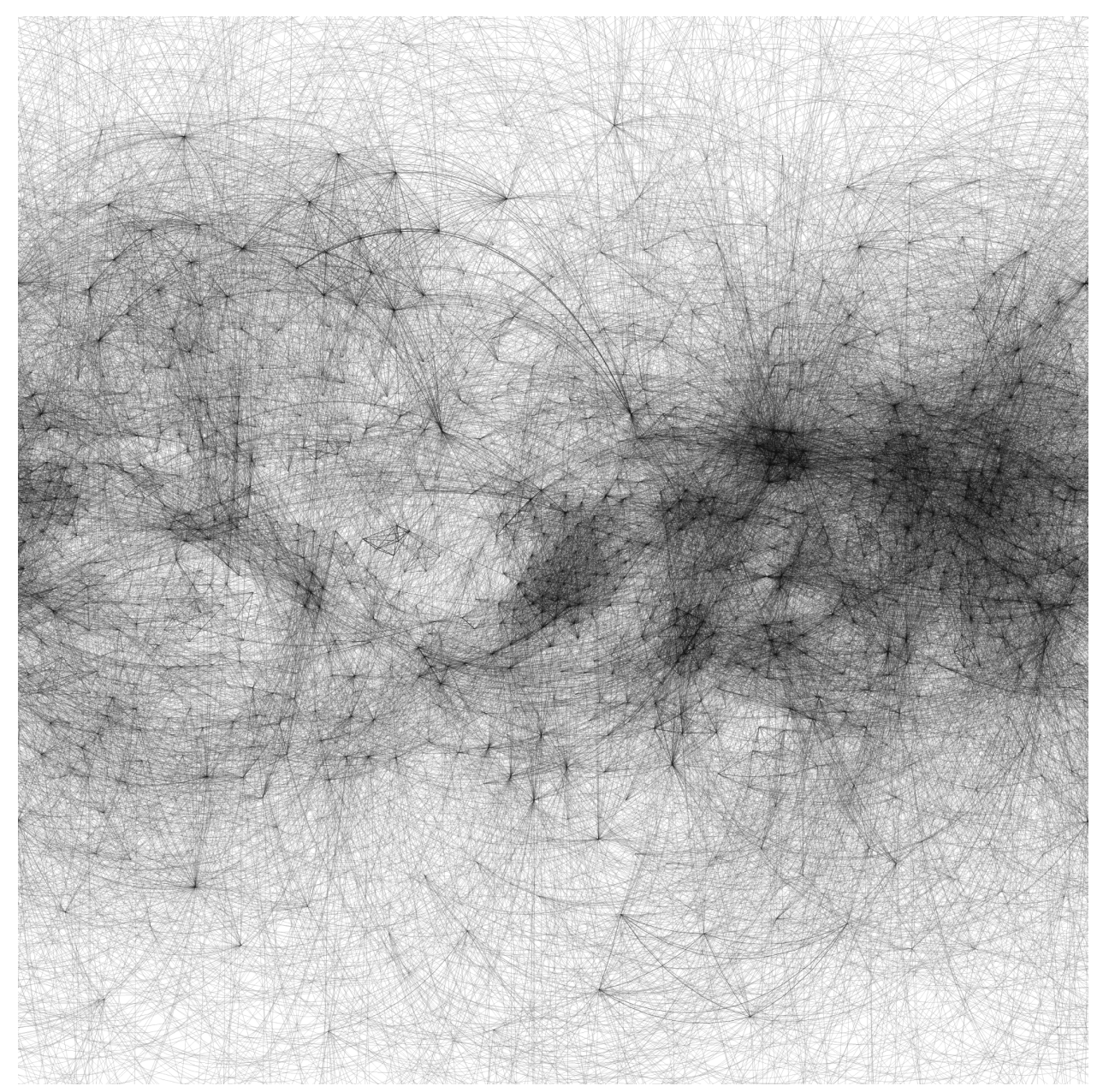

Fig. 4-La projection de Mercator appliquée à un réseau montre clairement comment la déformation de l'espace est reflétée par la longueur et la courbure des bords. Pour comprendre l'espace, il faut avant tout que le processus de conception soit clair.

La zone foncée de la projection Mercator montre comment le réseau occupe principalement la zone correspondant à l'équateur, ce qui permet une continuité horizontale. Cependant, d'autres projections ont des caractéristiques différentes comme la projection quinconciale de Charles Peirce ${ }^{19}$. Au xviii ${ }^{\text {e }}$ siècle, il était assez courant pour les étudiants et les universitaires de s'exercer à la cartographie ; Charles Peirce, qui est internationalement connu pour ses études de pragmatisme, a également contribué à la cartographie avec sa propre projection. La projection quinconciale est particulièrement pertinente dans ce contexte, car elle peut être carrelée dans toutes les directions (voir figure 5). Sa continuité s'étend à la fois dans les directions horizontale et verticale, créant un espace continu au Nord, à l'Est, au Sud et à l'Ouest. Contrairement à la projection de Mercator, la projection quinconciale conserve une grande précision aux pôles, ce qui en fait un outil parfait pour les expéditions arctiques naviguant dans l'hémisphère nord. Le

\footnotetext{
${ }^{19}$ Krämer, Sybille, and Christina Ljungberg, eds. 2017. Thinking with Diagrams: The Semiotic Basis of Human Cognition. Semiotics, Communication and Cognition [SCC], volume 17. Boston and Berlin: de Gruyter Mouton.
} 
pôle Nord est, en effet, exactement au centre de la carte tandis que le pôle Sud est réparti aux quatre coins. En regardant la figure 6, il est facile de remarquer comment les nœuds sont massés sur les diagonales de l'image. Plus difficile à lire, dans la même figure, est la déformation de l'espace qui est indiquée par les lignes courbes qui dessinent une forme en double $\mathrm{S}$ sur les diagonales. Comme nous l'avons dit précédemment, ces lignes sont droites sur la surface sphérique ; en gardant cela à l'esprit, il sera plus facile de percevoir la déformation spatiale du globe.

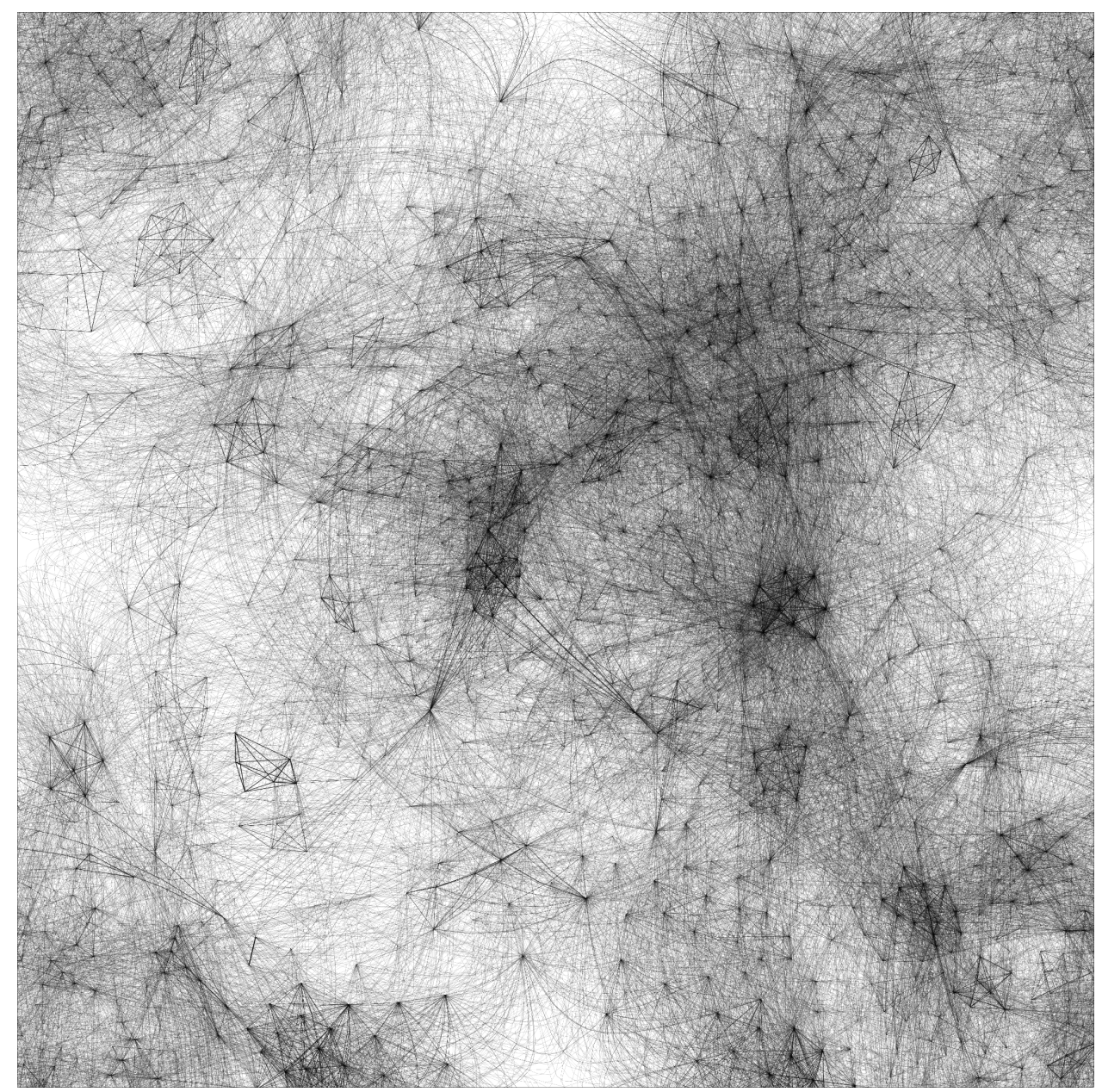

Fig. 5 - La projection quinconciale de Charles Peirce peut être carrelée dans toutes les directions. Cette caractéristique rend la disposition du réseau particulièrement intéressante, car la continuité de l'espace est garantie à la fois verticalement et horizontalement. Les zones de déformations majeures sont situées au milieu.

Nombreuses sont les projections cartographiques qui ont été conçues au fil du temps. Chacune d'entre elles permet au spectateur de voir différents aspects de la Terre, ce qui signifie également l'exploration de différentes formes dans ce contexte. Les projections orthographiques (figure 3) de Mercator (figure 4) et quinconciale (figure 5) sont les plus pertinentes en matière de 
transformation spatiale et de continuité. Pour autant, la bibliothèque D3 permet d'explorer des projections plus inhabituelles, que nous ne pouvons présenter ici : le papillon de Waterman, les coniques à aire égale, dodécaédrique, icosaédrique et tétraédrique de Lee.

\section{CONCLUSION}

Notre critique fictive contre la conspiration des réseaux plats est une façon de montrer comment les visualisations de réseaux peuvent encore progresser en termes de conception graphique depuis leur première apparition dans les sociogrammes de Jacob L. Moreno ${ }^{20}$. Bien que de nombreuses tentatives aient été faites dans l'espace tridimensionnel, personne n'avait jamais considéré les réseaux sphériques comme utiles pour la démocratisation de l'espace, et encore moins n’avait jamais osé appliquer la projection géographique aux réseaux mêmes.

Même si aujourd'hui le débat scientifique porte principalement sur l'éthique et la vie privée des données, le groupe d'universitaires concernés par la manière dont les données sont présentées est encore trop restreint. Mark Monmonier ${ }^{21}$, par exemple, a publié deux ouvrages sur la manière dont les graphiques peuvent véhiculer de fausses significations. Dans un moment historique où la quantification personnelle augmente de manière exponentielle, il est nécessaire de prendre soin non seulement du traitement des données, mais aussi de la manière dont les informations sont présentées.

L'étude de cas de la conférence en humanités numériques est un exemple de la manière dont les visualisations de données créées à partir de données personnelles peuvent créer des préjugés et conduire à des sentiments de discrimination. Aujourd'hui plus qu'hier, les concepteurs ont la responsabilité de présenter les données personnelles de la manière la plus appropriée. L'objectif de cet article est enfin d'encourager la discussion entre les concepteurs et les universitaires, en leur faisant prendre conscience de la responsabilité de développer des lignes directrices pour afficher les individus et les communautés de manière équitable, en travaillant sur des expériences et des observations simples comme celle-ci.

Je remercie pour le soutien à cette recherche Kurt Fendt et l'Active Archives Initiative (MIT), Jeffrey Schnapp et le Metalab (Harvard). Des remerciements vont également à José Higuera (Université de Porto), Alberto Romele (Université de Lille), Stéphane Vial (Université du Québec à Montréal), Boris Beaude et Ogier Maitre (Université de Lausanne) qui ont soutenu l'idée du projet. Enfin, tous mes remerciements à Philippe Rivière, qui a si brillamment appliqué la projection géographique aux réseaux.

\footnotetext{
${ }^{20}$ Moreno, Jacob L. 1934. Who Shall Survive? Washington: Nervous and Mental Disease Publishing Co.

${ }^{21}$ Monmonier, Mark S. 1991. How to Lie with Maps. Chicago: University of Chicago Press.
} 